
allemande

50-1 | 2018

Regards franco-allemands sur la justice dans la construction européenne | Les élections législatives de 2017 en Allemagne et en Autriche

\title{
Les élections législatives du 24 septembre 2017 et l'interminable avènement de la quatrième grande coalition
}

\author{
Michel Fabréguet
}

\section{OpenEdition}

Journals

\section{Édition électronique}

URL : https://journals.openedition.org/allemagne/643

DOI : 10.4000/allemagne.643

ISSN : 2605-7913

Éditeur

Société d'études allemandes

\section{Édition imprimée}

Date de publication : 30 juin 2018

Pagination : 91-104

ISSN : 0035-0974

\section{Référence électronique}

Michel Fabréguet, «Les élections législatives du 24 septembre 2017 et l'interminable avènement de la quatrième grande coalition », Revue d'Allemagne et des pays de langue allemande [En ligne], 50-1 | 2018, mis en ligne le 30 juin 2019, consulté le 02 juin 2022. URL : http://journals.openedition.org/allemagne/ 643 ; DOI : https://doi.org/10.4000/allemagne.643 


\title{
Les élections législatives du 24 septembre 2017 et l'interminable avènement de la quatrième grande coalition
}

\author{
- Michel Fabréguet*
}

\section{La campagne électorale (2016-2017)}

Un sondage publié par le Bild-Zeitung du mardi 10 mai 2016 sur les intentions de vote des Allemands dans la perspective des élections législatives de septembre 2017 créditait l'Union de 30,5 \% des voix, le SPD de 19,5 \% et l'Alternative pour l'Allemagne (AfD) de $15 \%^{(1)}$. Au cours de l'année 2015 , au cœur de la crise des réfugiés, alors que la plupart des pays d'Europe centrale érigeaient des barrières contre l'afflux des étrangers, l'Allemagne avait accueilli 890000 demandeurs d'asile. Le 31 août 2015, Angela Merkel avait employé pour la première fois la formule «Wir schaffen das» (Nous y arriverons). En 2016, l'Allemagne ouvrait encore ses portes à 280000 réfugiés. L'hospitalité libérale de la chancelière en matière d'immigration faisait alors l'objet des plus vives critiques, au moment même où cette question dominait le débat politique. À l'occasion de son congrès de Stuttgart, l'AfD avait proclamé que l'islam ne faisait pas partie de l'Allemagne et qu'il fallait interdire les minarets, les appels des muezzins et le voile intégral, alors que les fondateurs du mouvement Pegida se trouvaient condamnés pour incitation à la haine, après avoir comparé les réfugiés à du «bétail» et à des «ordures». "Plus émotionnelle que rationnelle», selon Michael Wolffsohn, la politique à l'égard des réfugiés renforçait les populistes, en sous-estimant aussi, selon Thilo Sarrazin, le coût énorme que représentaient pour les contribuables allemands, 21,7 milliards d'euros en 2016 et 21,3 milliards en 2017, les «migrations de masse incontrôlées » ${ }^{(2)}$. Ces critiques étaient

* Professeur à l'Institut d'études politiques, Université de Strasbourg.

1 Frédéric Lemaître, «En Allemagne les partis de gouvernement plongent dans les sondages. Conservateurs et sociaux-démocrates sont fragilisés alors que l'extrême droite progresse», Le Monde du 13 mai 2016.

2 Philip Plickart (éd.), Merkel. Eine kritische Bilanz, Munich, Finanz Buch Verlag, 2017, p. 156-159 et 169-174. 
relayées au sein de l'Union et de la coalition gouvernementale plus particulièrement par la CSU bavaroise. La crise des réfugiés avait attisé les tensions entre la chancelière et la CSU, qui exigeait la fin de la «culture de la bienvenue» et demandait que l'État fédéral assure son rôle de protecteur des frontières nationales en limitant à 200000 le nombre des réfugiés accueillis chaque année. Mécontente de ne pas être assez prise en considération par Angela Merkel, la CSU laissait planer l'hypothèse d'une dissidence à l'occasion des prochaines élections générales ${ }^{(3)}$ : cette forme de chantage avait déjà été brandie quarante ans plus tôt à la suite des élections législatives de 1976, lorsque les élus de la CSU de Franz Josef Strauß avaient opté pour la formation d'une fraction autonome au sein du Bundestag, avant que la menace de la CDU de venir s'implanter en Bavière n'y mette rapidement un terme. À la fin de l'été 2016, la question de la politique à l'égard des réfugiés dominait l'actualité et contribuait à affaiblir l'autorité de la chancelière. Mise en cause dans son propre parti, Angela Merkel pâtissait également des reculs successifs de la CDU à l'occasion des différents scrutins régionaux. En mars 2016, la CDU avait perdu 2,7 \% des voix en Saxe-Anhalt, 3,4 \% en RhénaniePalatinat et jusqu'à $12 \%$ dans le Land de Bade-Wurtemberg. Le 4 septembre, en recul de $4 \%$, la CDU fut devancée par l'AfD en Mecklembourg-Poméranie occidentale et le 18 septembre la CDU n'obtint que $17,6 \%$ des voix à Berlin, en recul de 5,7 \% par rapport à 2011, devancée par le SPD à 21,6\%, en fort recul également, avec lequel l'Union avait gouverné la capitale allemande pendant cinq ans. À la suite du scrutin berlinois, Eberhard Diepgen, dernier maire chrétien-démocrate de Berlin de 1991 à 2001, avait justement réclamé que la CDU «se positionne plus à droite qu'elle ne l'est à présent ${ }^{(4)}$.

Critiques à l'égard d'une chancelière à laquelle ils reprochaient une orientation par trop «sociale-démocrate», les conservateurs n'avaient cependant pas d'autre alternative que de se ranger derrière elle dans la perspective des prochaines élections générales. Rejetant le spectre d'une coalition rouge-rouge-verte, la CSU investit Angela Merkel comme candidate à la chancellerie lors de son congrès de Munich les 4 et 5 novembre $2016^{(5)}$ et la CDU reconduisit la chancelière dans ses fonctions de présidente du parti à l'occasion du congrès d'Essen les 6 et 7 décembre $2016^{(6)}$. Dans l'intervalle, Angela Merkel avait officialisé sa candidature à un quatrième mandat, après avoir annoncé, dans un désir de stabilité, son soutien à la candidature de son ministre des Affaires étrangères, le social-démocrate Frank-Walter Steinmeier, à la prochaine élection présidentielle prévue pour le 12 février $2017^{(7)}$.

Face à la chancelière sortante, et en dépit des difficultés auxquelles elle s'était trouvée confrontée tout au long de l'année 2016, le SPD, partenaire de l'Union au sein de la troisième grande coalition, se trouvait confronté à un problème de leadership.

3 F. Lemaître, «En Allemagne les partis de gouvernement plongent dans les sondages» (note 1).

4 Thomas WiEder, «À Berlin, nouveau revers pour Angela Merkel. La chancelière prend ses distances avec la formule controversée sur l'accueil des réfugiés», Le Monde du 20 septembre 2016.

5 Thomas WiEder, «La CSU se range derrière Angela Merkel, malgré tout », Le Monde du 8 novembre 2016.

6 Thomas WiEder, "Angela Merkel donne des gages à la CDU sans se renier», Le Monde du 8 décembre 2016.

7 Thomas WiEder, «Merkel se résout au choix du SPD pour la présidence», Le Monde du 16 novembre 2016. 
Vice-chancelier et ministre fédéral de l'Économie, Sigmar Gabriel apparaissait comme le concurrent naturel d'Angela Merkel mais, peu populaire dans l'opinion et contesté par l'aile gauche du SPD, il souffrait d'un manque de charisme évident: sous sa direction, le SPD n'était crédité que de $20 \%$ des intentions de vote, très largement distancé par des partis de l'Union. Le 24 janvier 2017, Sigmar Gabriel annonça qu'il prendrait la succession de Frank-Walter Steinmeier à la tête du ministère des Affaires étrangères et qu'il ne se présenterait pas à la chancellerie, en s'effaçant au profit de Martin Schulz, jusqu'alors président en exercice du Parlement européen, qui avait annoncé à la fin de l'année 2016 sa candidature aux élections législatives allemandes de $2017^{(8)}$. L'annonce de la candidature de Martin Schulz suscita immédiatement une dynamique au profit du SPD : l'écart dans les intentions de vote entre les deux partis populaires qui était de 17 points au profit de l'Union au début du mois de janvier 2017 se resserra à quelques points au début du mois de février, et le SPD devança même l'Union d'un point dans un sondage de l'institut Emnid publié par le Bild-Zeitung du 19 février, ce qui n'était plus arrivé depuis $2006^{(9)}$. À la différence de Sigmar Gabriel, Martin Schulz bénéficiait d'un fort capital de sympathie dans l'opinion: homme issu du peuple qui n'avait pas suivi d'études supérieures, il apparaissait aussi comme un homme nouveau sur la scène politique allemande, après vingt années passées au Parlement européen, non compromis par l'exercice du pouvoir à Berlin dans les deux précédents gouvernements de grande coalition. Il avait également su prendre ses distances avec Gerhard Schröder et «l'Agenda 2010 ». La dynamique penchait donc nettement au profit du SPD, au moment où Steinmeier fut élu président de la République le 12 février 2017 par 931 voix des 1260 grands électeurs de l'Assemblée fédérale, avec le soutien de l'Union, du SPD, des Verts et du $\operatorname{FDP}^{(10)}$. Le 19 mars 2017, $100 \%$ des participants au congrès du SPD à Berlin investirent Martin Schulz à la présidence du parti ${ }^{(11)}$. Mais l'engouement de l'opinion, que la «flambée sondagière» du début de l'année 2017 avait enregistré, demeura purement conjoncturel. Trois élections régionales au printemps 2018 vinrent rapidement inverser la tendance et démentir les illusions des sociaux-démocrates: en Sarre le 26 mars où la candidate du SPD Anke Rehlinger ne tira aucun profit de «l'effet Schulz» et fut nettement devancée par la candidate de la CDU, Annegret Kramp-Karrenbauer, dans le Schleswig-Holstein le 7 mai 2018 et surtout en Rhénanie du Nord-Westphalie, le 14 mai 2018, où à la suite d'une campagne de la CDU assez efficace sur le thème de la sécurité, après les agressions sexuelles commises à Cologne le 31 décembre 2015, le SPD en recul de 7,5 points par rapport à 2012 obtint son plus mauvais résultat depuis 1947 et perdit son bastion au profit d'un candidat conservateur à la faible notoriété(12). À

8 Cécile Ducourtieux, «Martin Schulz va quitter le Parlement européen », Le Monde du 25 novembre 2016; Cécile Ducourtieux et Thomas Wieder, "Schulz sera le candidat du SPD contre Merkel», Le Monde du 26 janvier 2017.

9 Thomas Wieder, «Schulz bouscule Merkel dans les sondages», Le Monde du 7 février 2017; Thomas WiEder, «Schultz peut-il gagner contre Merkel?», Le Monde du 24 février 2017.

10 Thomas Wieder, «Un anti-Trump à la présidence de l’Allemagne», Le Monde du 14 février 2017.

11 Thomas Wieder, «Le SPD euphorique autour de son candidat Schulz», Le Monde du 21 mars 2017.

12 Thomas Wieder, «En Sarre, Merkel et la CDU résistent à l'effet Schulz», Le Monde du 28 mars 2017; Thomas WiEDER, "Sévère défaite des sociaux-démocrates allemands dans le Schleswig-Holstein", Le Monde du 9 mai 2017; Thomas Wieder, «En Allemagne, le SPD dans la tourmente», Le Monde du 16 mai 2017. 
partir de la fin du mois d'avril 2017, la courbe des intentions de vote s'inversa et le SPD se retrouva à nouveau assez sensiblement distancé par les partis de l'Union. À partir de la fin du printemps de l'année 2017, l'issue des élections générales de septembre 2017 ne laissa plus guère planer de doutes, alors même que les partis n'avaient pas encore fait connaître leurs programmes. Dans un contexte international difficile, qui suscitait des inquiétudes dans l'opinion, à la suite de la victoire de Trump à l'élection présidentielle américaine, des doutes sur l'avenir de l'Europe avec le Brexit et des tensions avec la Turquie et la Russie, l'expérience d'Angela Merkel jouait en sa faveur et la chancelière allemande semblait devoir être épargnée par le «dégagisme» qui emportait dans le même temps les autres responsables européens de premier plan ${ }^{(13)}$. Le 25 juin 2017, le congrès de Dortmund du SPD valida le programme d'un parti qui cherchait à se démarquer des orientations de ses partenaires de coalition, en matière fiscale en faisant passer de $42 \%$ à $45 \%$ le taux d'imposition des revenus supérieurs à 76200 euros, en matière de défense, en refusant de prendre en considération l'objectif fixé par l'OTAN des $2 \%$ du PIB consacré aux dépenses militaires, en matière de politique européenne ou encore sur le terrain du mariage homosexuel ${ }^{(14)}$. Mais sur le fond, les différences avec le programme de l'Union restaient limitées et il était difficile au SPD d'attaquer frontalement un partenaire avec lequel il venait de gouverner pendant quatre ans. Sur le dossier du mariage pour tous, Angela Merkel fit à nouveau preuve de son habileté manœuvrière coutumière: le SPD, les Verts et le FDP ayant fait savoir qu'ils ne signeraient pas un contrat de coalition qui ne mentionnerait pas son adoption, la chancelière fit voter par le Bundestag le 30 juin 2017, au pas de charge après un débat de quarante minutes, une loi instituant les unions homosexuelles, adoptée par 393 voix contre 227 avec l'appui du SPD, des Verts, de Die Linke et d'un tiers des députés de l'Union, Angela Merkel se prononçant elle-même contre le projet de loi, mais sans prendre de grands risques, $75 \%$ des Allemands étant alors favorables au mariage homosexuel ${ }^{(15)}$.

À la fin de l'été 2017, le SPD et Martin Schulz n'étaient pas parvenus à imposer leurs thèmes de campagne face aux conservateurs, alors que les Verts semblaient devenus inaudibles face à une chancelière qui les avait dépouillés de leur programme. Le débat télévisé du 3 septembre entre Angela Merkel et Martin Schulz fut trop consensuel pour permettre au candidat social-démocrate d'inverser les rapports de force, ce dernier donnant même le sentiment de ne plus croire en sa victoire. Dans les dernières semaines de la campagne, c'est en fait l'extrême droite de l'AfD, jusqu'alors en perte de vitesse par suite de ses divisions internes et de la moindre place occupée dans le débat public par la question des réfugiés, qui chercha à incarner la véritable opposition au pouvoir en place par la virulence de ses attaques contre la chancelière, en particulier dans les nouveaux Bundesländer ${ }^{(16)}$. À la veille de l'élection, la chancelière était pratiquement sûre d'être réélue, mais l'incertitude demeurait quant à la configuration de la future

13 Thomas Wieder, «Pourquoi Angela Merkel est-elle toujours aussi populaire», Le Monde des 18 et 19 juin 2017.

14 Thomas WiEdER, «Face à Merkel, Schulz veut y croire encore», Le Monde du 27 juin 2017.

15 Thomas Wieder, "Angela Merkel prête à faire adopter le mariage pour tous", Le Monde du 29 juin 2017; Thomas WiEDER, «Les députés allemands adoptent le mariage pour tous, sans Merkel», Le Monde du $1^{\text {er }}$ juillet 2017.

16 Thomas Wieder, «La campagne au canon de l'AfD contre Merkel», Le Monde du 12 septembre 2017. 
coalition gouvernementale. L'opinion était divisée et surtout très sceptique: selon un sondage, 51,3 \% des personnes interrogées pensaient qu'aucune coalition envisageable n'était appropriée, $24,1 \%$ se prononçaient en faveur de la coalition alternative de gauche rouge-rouge-verte, $9,4 \%$ se déclaraient favorables à la reconduction de la grande coalition, 9,3\% seulement à l'expérimentation d'une coalition jamaïcaine noire-jauneverte $^{(17)}$ et 5,9 \% se prononçaient en faveur d'une coalition entre la CDU et les Verts ${ }^{(18)}$.

\section{Le résultat des élections législatives du 24 septembre 2017}

\begin{tabular}{|l|l|l|}
\hline Nombre d'électeurs inscrits & & 61688485 \\
\hline Votants & $76,2 \%$ & 46976341 \\
\hline Secondes voix valables & $99,0 \%$ & 46515492 \\
\hline
\end{tabular}

Résultat des élections législatives du 24 septembre $2017^{(19)}$

\begin{tabular}{|c|c|c|c|c|}
\hline Partis & $\begin{array}{c}\% \\
\text { Premières } \\
\text { voix }\end{array}$ & $\begin{array}{c}\% \\
\text { Secondes } \\
\text { voix }\end{array}$ & $\begin{array}{l}\text { Gains/pertes } \\
2017-2013 \text { en } \\
\text { secondes voix }\end{array}$ & $\begin{array}{c}\text { Nombre de } \\
\text { secondes voix }\end{array}$ \\
\hline CDU & $30,2 \%$ & $26,8 \%$ & $\begin{array}{r}-2474221 \\
(-7,4 \%)\end{array}$ & 12447656 \\
\hline SPD & $24,6 \%$ & $20,5 \%$ & $\begin{array}{r}-1712834 \\
(-5,2 \%)\end{array}$ & 9539381 \\
\hline Die Linke & $8,6 \%$ & $9,2 \%$ & $\begin{array}{r}+541571 \\
(+0,6 \%)\end{array}$ & 4297270 \\
\hline Les Verts & $8,0 \%$ & $8,9 \%$ & $\begin{array}{r}+464343 \\
(+0,5 \%)\end{array}$ & 4158400 \\
\hline CSU & $7,0 \%$ & $6,2 \%$ & $\begin{array}{r}-373881 \\
(-1,2 \%)\end{array}$ & 2869688 \\
\hline FDP & $7,0 \%$ & $10,7 \%$ & $\begin{array}{r}+2915916 \\
(+6,0 \%)\end{array}$ & 4999449 \\
\hline AfD & $11,5 \%$ & $12,6 \%$ & $\begin{array}{r}+3821130 \\
(+7,9 \%)\end{array}$ & 5878115 \\
\hline Autres partis & $3,1 \%$ & $5 \%$ & $\begin{array}{r}-4533906 \\
(-10,7 \%)\end{array}$ & 2325533 \\
\hline dont Pirates & $0,2 \%$ & $0,4 \%$ & $\begin{array}{r}-785701 \\
(-1,8 \%)\end{array}$ & 173476 \\
\hline dont NPD & $0,1 \%$ & $0,4 \%$ & $\begin{array}{r}-384808 \\
(-0,9 \%)\end{array}$ & 176020 \\
\hline $\begin{array}{r}\text { dont } \\
\text { Électeurs libres }\end{array}$ & $1,3 \%$ & $1,0 \%$ & $\begin{array}{l}+39315 \\
(+0,0 \%)\end{array}$ & 463292 \\
\hline
\end{tabular}

17 Les couleurs de la CDU (noir), des libéraux-démocrates (jaune) et des Verts permettent de composer le drapeau de la Jamaïque d'où l'expression de coalition jamaïcaine pour désigner la coalition de ces trois partis.

18 Bundestagswahl 2017: Ergebnis, Sitzverteilung, Koalitionen, Kosten, https://www.wahlen-deutschland. com/ergebnis/

19 Der Bundeswahlleiter, Wahl zum 19. deutschen Bundestag am 24. September 2017, Heft 3: Endgültige Ergebnisse nach Wahlkreisen, p. 9. 
Les élections législatives du dimanche 24 septembre 2017 ont confirmé l'éclatement du paysage politique allemand annoncé par les instituts de sondages avec le maintien dans la $19^{\mathrm{e}}$ législature des quatre fractions sortantes, le retour du FDP et l'entrée dans le nouveau Bundestag de l'AfD. Mais la surprise a consisté dans le net revers des partis populaires associés dans la troisième grande coalition, plus bas dans les urnes que ne prévoyaient les instituts de sondages, en particulier les partis de l'Union, alors que le score de l'AfD dépassait sensiblement les estimations les plus optimistes à la veille du scrutin. Les partis de la grande coalition sortante ont perdu ensemble 4560936 secondes voix, les pertes les plus importantes affectant les deux partis de l'Union, 2848102 secondes voix soit un recul de 8,6 \%, le SPD perdant pour sa part 1712834 voix soit un recul de 5,2\%. Avec moins de $33 \%$ des secondes voix, les deux partis de l'Union ont enregistré leur deuxième plus mauvais résultat de toute l'histoire électorale de la République fédérale d'Allemagne, en étant pratiquement ramenés à l'étiage des $31 \%$ obtenus le 14 août 1949 lors du premier scrutin législatif de la RFA également caractérisé par une forte dispersion des voix dans le cadre d'un processus de transition avec l'ancien système des partis de la République de Weimar. Ne parvenant à mobiliser qu'un faible contingent de 380000 électeurs non votants lors du précédent scrutin du 22 septembre 2013, l’Union a enregistré ses plus fortes pertes au profit du FDP, quelque 1360000 électeurs chrétiens-démocrates de 2013 ayant accordé en 2017 leurs secondes voix au $\mathrm{FDP}^{(20)}$, dans un mouvement inverse à celui de 2013 qui avait largement contribué à priver les libéraux-démocrates d'une représentation parlementaire dans la $18^{\mathrm{e}}$ législature. Mais les partis de l'Union ont également perdu 980000 électeurs au profit de l'AfD, en particulier dans les nouveaux Bundesländer de l'Allemagne de l'Est et dans les Länder de l'Allemagne du Sud. Si les pertes du SPD restent inférieures à celles de leurs partenaires de coalition, le résultat n'en reste pas moins désastreux pour le parti, tombé en dessous des calamiteux $23 \%$ de septembre 2009. Il s'agit en fait du plus faible score du SPD dans l'histoire électorale de la RFA depuis 1949, et sur la longue durée de l'histoire électorale allemande contemporaine, les $20,5 \%$ du 24 septembre 2017 sont à rapprocher des 20,4\% obtenus par le SPD à l'occasion du scrutin du 6 novembre 1932, dans le contexte traumatique de la crise de la République de Weimar. C'est l'étiage le plus bas du parti dans l'histoire électorale allemande depuis la "préhistoire» du Reich bismarckien. Plus préoccupant encore pour le SPD: si les pertes des partis de l'Union restent principalement cantonnées à la droite et au centre-droit de l'échiquier politique allemand, le SPD a perdu pour sa part presque autant d'électeurs sur sa droite que sur sa gauche, 470000 au profit de l'AfD, 450000 au profit du FDP, 430000 au profit de Die Linke et 380000 au profit des Verts, signe évident de la fragilisation et de la perte d'identité d'un parti attaqué sur tous ses flancs ${ }^{(21)}$.

Le score de l'AfD, avec 12,6 \% des secondes voix, a constitué l'événement médiatique de la soirée du 24 septembre et suscité l'inquiétude des commentateurs, qui n'attendaient pas les souverainistes allemands à un aussi haut niveau. Resté à la porte du Bundestag le 22 septembre 2013 par suite d'un déficit de 132752 secondes voix pour

20 Frankfurter Allgemeine Zeitung (FAZ), Dienstag, 26. September 2017, n²24, p. 9. Ein Beitrag der FAZ für Bundestagwahl 2017. Tous les transferts de voix entre les partis mentionnés dans cette seconde partie sont issus de la même source.

21 Le phénomène a concerné également, mais dans de moindres proportions, les Verts qui ont perdu 170000 électeurs au profit de Die Linke et 110000 électeurs au profit du FDP. 
atteindre le seuil des $5 \%{ }^{(22)}$, le nouveau parti, aimanté par le mouvement islamophobe Pegida, a réussi son entrée dans le $19^{\mathrm{e}}$ Bundestag après avoir obtenu 7,1 \% des voix lors des élections européennes de $2014^{(23)}$. Le succès des populistes souverainistes est lié à leur pouvoir d'attraction sur les autres électorats populaires, à la manière d'un parti «attrape tout»: l'AfD a attiré 1200000 électeurs qui s'étaient abstenus en septembre 2013, mais aussi 980000 électeurs de l'Union, 470000 électeurs sociaux-démocrates, 400000 électeurs de Die Linke et elle a siphonné l'électorat du NPD condamné à une quasi-disparition. De manière tout aussi significative, son pouvoir d'attraction sur les électorats écologistes et libéraux-démocrates, culturellement et socialement les plus favorisés, est resté marginal ${ }^{(24)}$. La percée de l'AfD a en partie occulté la «résurrection» du FDP, éliminé du précédent Bundestag pour un déficit de 102819 secondes voix, qui est parvenu sous l'impulsion de Christian Lindner à reconquérir un électorat conservateur qui lui avait fait défaut en 2013, mais également à conquérir les suffrages de 700000 abstentionnistes de 2013 et de plus d'un demi-million d'électeurs de la gauche sociale-démocrate ou écologiste.

Répartition des secondes voix entre les principaux partis entre l'Ouest et l'Est de l'Allemagne ${ }^{(25)}$

\begin{tabular}{|c|c|c|c|c|c|}
\hline \multicolumn{2}{|c|}{$\begin{array}{c}\text { Allemagne } \\
\text { dans son ensemble }\end{array}$} & \multicolumn{2}{|c|}{$\begin{array}{c}\text { Ouest } \\
\text { (avec Berlin-Ouest) }\end{array}$} & \multicolumn{2}{|c|}{$\begin{array}{c}\text { Est } \\
\text { (avec Berlin-Est) }\end{array}$} \\
\hline CDU & $26,8 \%$ & CDU & $26,6 \%$ & CDU & $27,6 \%$ \\
\hline SPD & $20,5 \%$ & SPD & $21,9 \%$ & AfD & $21,9 \%$ \\
\hline AfD & $12,6 \%$ & FDP & $11,5 \%$ & Die Linke & $17,8 \%$ \\
\hline FDP & $10,7 \%$ & AfD & $10,7 \%$ & SPD & $13,9 \%$ \\
\hline Die Linke & $9,2 \%$ & Les Verts & $9,8 \%$ & FDP & $7,5 \%$ \\
\hline Les Verts & $8,9 \%$ & CSU & $7,5 \%$ & Les Verts & $5 \%$ \\
\hline CSU & $6,2 \%$ & Die Linke & $7,4 \%$ & & \\
\hline
\end{tabular}

Le scrutin du 24 septembre 2017 a confirmé la permanence des diversités régionales selon les deux axes ouest/est et nord/sud qui structurent toujours l'expression politique des Allemands. Sur le territoire de l'ex-Allemagne de l'Ouest d'avant 1989 se maintient un système associant les deux grands partis populaires, mais très affaiblis par rapport à la précédente consultation où ils rassemblaient encore $69,6 \%$ des secondes voix alors qu'ils n'en représentent plus désormais que $56 \%$, à quatre petits partis dont l'audience individuelle s'échelonne de 7,4 \% à 11,5\%. Sur le territoire de l'ex-RDA, la hiérarchie de l'ancien système tripartite CDU-Die Linke-SPD est bouleversée par la percée de l'AfD de $5,8 \%$ à $21,9 \%$ des secondes voix qui vient conquérir la seconde place au détriment de Die Linke, en fort recul de 4,9\% mais qui parvient tout de même à se maintenir

22 Michel FAbréguet, «Les élections législatives du 22 septembre 2013 et la formation de la troisième grande coalition", Revue d'Allemagne et des pays de langue allemande, 46/1, janvier-juin 2014, p. 145.

23 Yves Camus et Nicolas Lebourg, Les droites extrêmes en Europe, Paris, Éditions du Seuil, 2015, p. 244.

24 En 2013 cependant, l'AfD avait attiré 430000 anciens électeurs du FDP.

25 Der Bundeswahlleiter, Wahl zum 19. deutschen Bundestag am 24. September 2017 (note 19), p. 326. 
dans son bastion historique de Berlin-Est, et du SPD rejeté à la quatrième place et qui peine désormais à l'Est à pouvoir prétendre encore au statut de parti populaire avec seulement $13,9 \%$ des secondes voix.

Mais en fonction des diversités régionales perceptibles sur le territoire de l'exAllemagne de l'Ouest selon l'axe nord/sud, trois grands sous-ensembles régionaux s'individualisent finalement assez nettement à l'issue du scrutin du 24 septembre 2017. Dans l'Allemagne du Nord-Ouest, composée des Länder du Schleswig-Holstein, de Hambourg, de Basse-Saxe, de Brême, de Rhénanie du Nord-Westphalie, de Hesse, de Rhénanie-Palatinat et de la Sarre, la CDU remporte 113 mandats directs et le SPD 55 , et par ailleurs la progression du FDP reste globalement supérieure et contient donc celle de l'AfD, même si les gains de l'AfD restent cependant légèrement supérieurs à ceux des libéraux-démocrates en Basse-Saxe, à Brême, en Hesse et dans la Sarre. Dans l'Allemagne du Sud par contre la totalité des mandats directs revient aux seuls partis de l'Union, 46 à la CSU en Bavière et 38 à la CDU dans le Bade-Wurtemberg, l'écologiste Cern Özdemir avec $29,7 \%$ des premières voix ne parvenant en particulier qu'à talonner le chrétien-démocrate Stefan Kaufmann à $32 \%$ dans la circonscription de Stuttgart I. À la différence de l'Allemagne du Nord-Ouest, la progression de l'AfD dans le Sud est partout supérieure à celle du FDP, la situation la plus nette se révélant en Bavière où l'AfD progresse de 632730 secondes voix, se positionnant ainsi au troisième rang sur le plan régional derrière la CSU et le SPD, le FDP gagnant pour sa part 417090 secondes voix tandis que la CSU perd plus de 10 points en recul de 373881 secondes voix ${ }^{(26)}$. Les nouveaux Bundesländer de l'ex-Allemagne de l'Est constituent le troisième sous-ensemble régional caractérisé par la percée de l'AfD au détriment de l'ensemble des partis de l'ancien système tripartite et du NPD, que ne contiennent pas, à la différence de l'Allemagne du Nord-Ouest, les progrès plus modestes du FDP à l'Est. La percée de l'AfD est partout spectaculaire avec des gains de $13 \%$ des secondes voix en Mecklembourg-Poméranie occidentale, de 14,2 \% dans le Brandebourg, de 15,4 \% en Saxe-Anhalt et de 16,5\% en Thuringe. La progression de l'AfD est la plus spectaculaire en Saxe où en gagnant plus d'un demi-million de secondes voix, soit une percée de 20,3\%, l'AfD se positionne au premier rang régional avec $27 \%$ des secondes voix, parvenant à devancer la CDU de plus de 4000 voix. Elle est en mesure d'obtenir 11 des 38 sièges de la représentation du Land au Bundestag, dont les 3 mandats directs de Bautzen I, de Görlitz et de Sächsische Schweiz-Ostergebirge, circonscription dans laquelle est élue Frauke Petry ${ }^{(27)}$, qui devait annoncer son départ de l'AfD dès le 26 septembre 2017.

Avec le retour du FDP et l'entrée de l'AfD dans le Bundestag, six fractions parlementaires sont ainsi en mesure de se constituer dans l'enceinte de la $19^{\mathrm{e}}$ législature, représentant $95 \%$ des secondes voix exprimées à l'occasion d'un scrutin où la participation a été en hausse de 5,1\% par rapport à la précédente consultation. Le nouveau Parlement peut ainsi échapper à la critique d'une insuffisante représentativité nationale qui avait pu être adressée à son prédécesseur, désigné à la suite d'une élection au cours de laquelle $28,5 \%$ des électeurs inscrits ne s'étaient pas déplacés et 15,8 \% des secondes voix exprimées n'étaient pas représentées dans le Bundestag. La situation présente est donc très satisfaisante du point de vue de l'éthique démocratique, même si elle va poser de réelles 
difficultés en vue de la constitution d'une nouvelle coalition gouvernementale stable, au regard justement de l'éclatement et de la dispersion de la représentation nationale. Elle témoigne finalement de l'équité et de la finesse de la loi électorale du double vote. Bien loin de figer et de rigidifier le système des partis, au risque d'aboutir à un divorce de plus en plus prononcé entre la représentation nationale et le pays réel, la loi électorale du double vote a permis au contraire au processus de formation de la représentation nationale d'épouser et de se calquer en permanence sur les évolutions de l'opinion publique sur la longue durée. Après avoir accompagné et encadré le processus de concentration des partis sur la scène politique ouest-allemande à l'époque de la Guerre froide, dans les années 1950 et 1960, la loi électorale allemande inchangée a été en mesure de permettre, depuis le début des années 1980, une restauration de la tradition allemande du multipartisme. Appréhendée dans le passé par les chercheurs au moyen des concepts aujourd'hui inopérants de camps ou de milieux, celle-ci vient de parvenir à son terme, vingt-huit ans après la réunification allemande, par l'admission dans l'enceinte du nouveau Bundestag de représentants d'une droite extrême populiste et souverainiste: pour la première fois depuis les premières élections d'août 1949, des représentants de l'extrême droite siègent à nouveau dans la chambre des députés qui est ainsi en mesure de représenter la quasitotalité des forces politiques du pays. Savoir si celles-ci seront en mesure d'en tirer profit en débattant véritablement entre elles de manière constructive et profitable à l'intérêt général constitue à vrai dire un autre débat.

Gains et pertes dans la répartition des sièges entre les partis lors de la $19^{\mathrm{e}}$ législature ${ }^{(28)}$

\begin{tabular}{|l|c|c|c|}
\hline Partis & $\begin{array}{c}\text { Nombre total } \\
\text { de sièges }\end{array}$ & $\begin{array}{c}\text { Mandats } \\
\text { directs }\end{array}$ & $\begin{array}{c}\text { Mandats de } \\
\text { liste }\end{array}$ \\
\hline CDU & $200(-55)$ & $185(-6)$ & $15(-49)$ \\
\hline SPD & $153(-40)$ & $59(+1)$ & $94(-41)$ \\
\hline Die Linke & $69(+5)$ & $5(+1)$ & $64(+4)$ \\
\hline Les Verts & $67(+4)$ & $1(0)$ & $66(+4)$ \\
\hline CSU & $46(-10)$ & $46(+1)$ & $0(-11)$ \\
\hline FDP & $80(+80)$ & 0 & $80(+80)$ \\
\hline AfD & $94(+94)$ & $3(+3)$ & $91(+91)$ \\
\hline Total & $709(+78)$ & $299(0)$ & $410(+78)$ \\
\hline
\end{tabular}

\section{La plus longue crise politique de l'histoire de la République fédérale d'Allemagne (septembre 2017-mars 2018)}

En dehors de l'AfD et du FDP, le résultat du 24 septembre 2017 ne pouvait contenter un grand nombre de personnes. Selon un sondage effectué le 25 septembre, $81,6 \%$ des Allemands demeuraient insatisfaits du résultat ${ }^{(29)}$. Angela Merkel, gagnée par l'usure du pouvoir, semblait a priori assurée d'être reconduite mais le visage de la future

28 Der Bundeswahlleiter, Wahl zum 19. deutschen Bundestag am 24. September 2017 (note 19), p. 332.

29 Bundestagswahl 2017: Ergebnis, Sitzverteilung, Koalitionen, Kosten (note 18). 
coalition gouvernementale demeurait incertain. Mathématiquement, en dehors d'une inconcevable coalition rouge-rouge-verte-jaune, deux options s'offraient sur la base des résultats: soit la reconduction d'une grande coalition assurée du soutien théorique de 399 députés sur 709 au Bundestag, représentant 53,4 \% du corps électoral, soit l'expérimentation d'une coalition jamaïcaine disposant de 393 élus et représentant $52,5 \%$ du corps électoral ${ }^{(30)}$. Mais dès l'annonce des résultats, Martin Schulz avait refusé la première hypothèse en déclarant «ce soir prend fin le travail avec la CDU et la $\mathrm{CSU}{ }^{(31)}$ et en assurant le lendemain que «jamais [il] n'entrerait dans un gouvernement avec $\mathrm{M}^{\text {me }}$ Merkel». Martin Schulz fut rapidement rejoint par Andrea Nahles, nouvelle présidente de la fraction sociale-démocrate au Bundestag, qui se prononça pour une opposition sans concessions aux conservateurs. La défaite historique du SPD ne lui laissait pas d'autres choix que le retour à l'opposition et le parti, confronté à une crise commune à l'ensemble des formations sociales-démocrates en Europe, se trouvait contraint d'engager une réflexion programmatique accompagnée d'un rajeunissement et d'une modernisation de son mode d'organisation. La courte victoire obtenue par le parti lors des élections régionales de Basse-Saxe le 15 octobre 2017 ne pouvait compenser la série des déconvenues électorales subies au cours de la même année ${ }^{(32)}$.

Devant l'impossibilité d'une reconduction de la grande coalition, la voie d'une coalition jamaïcaine restait la seule envisageable. Elle était expérimentée depuis le printemps dans le Schleswig-Holstein. Mais sa mise en œuvre à l'échelon fédéral demeurait très incertaine tant les désaccords entre les partis concernés restaient nombreux: sur l'environnement, la sécurité et l'Europe les Verts et les libéraux-démocrates s'opposaient ouvertement, le FDP refusant le budget de la zone euro réclamé par les Verts, lesquels demandaient la fermeture des centrales à charbon défendues par le FDP et manifestaient leur hostilité à la mise en place d'un seuil annuel pour l'accueil des demandeurs d'asile ${ }^{(33)}$. Le FDP posait par ailleurs des conditions pour une alliance avec les conservateurs et revendiquait le ministère fédéral des Finances détenu par Wolfgang Schäuble. Affaiblie par le résultat du scrutin, Angela Merkel sacrifia son ministre des Finances dont l'élection à la présidence du Bundestag fut annoncée à la fin du mois de septembre. Et surtout elle fut dans l'obligation de se plier aux exigences de la CSU en annonçant la limitation à 200000 du nombre des réfugiés accueillis chaque année ${ }^{(34)}$. Avant d'entamer des négociations avec ses futurs partenaires, la chancelière dut ressouder les liens avec les membres de sa propre famille et accéder, lors d'une entrevue à Berlin le 8 octobre, aux exigences de la CSU, menacée par la montée en puissance de l'AfD en Bavière de perdre la majorité absolue à l'occasion des prochaines élections régionales de l'automne 2018, décision qui ne pouvait que compliquer les négociations à venir avec les Verts.

30 Ibid.

31 Thomas Wieder, «Angela Merkel gagnée par l'usure du pouvoir», Le Monde du 26 septembre 2017.

32 Thomas Wieder, «La crise profonde du SPD», Le Monde du 19 octobre 2017.

33 Thomas Wieder, «Angela Merkel gagnée par l'usure du pouvoir», Le Monde du 26 septembre 2017.

34 Thomas Wieder, «Schäuble, première victime de revers de Merkel», Le Monde du 29 septembre 2017; Thomas Wieder, "Merkel cède aux exigences de son allié bavarois sur les migrants", Le Monde du 10 octobre 2017. 
La conduite des conversations exploratoires entre les futurs partenaires, très fortement médiatisée sur la scène publique, ne facilita guère les procédures d'arbitrage et de conciliation. Finalement, c'est une déclaration unilatérale du président du FDP, Christian Lindner, le dimanche 19 novembre 2017, qui officialisa l'échec des négociations. La situation était inédite en Allemagne: deux mois après les élections, l'incertitude restait entière sur les contours de la future majorité gouvernementale. De fortes pressions s'exercèrent alors sur la direction du SPD. Le président de la République appela le 23 novembre les partis susceptibles de former un gouvernement à la "responsabilité ». Le 30 novembre, le président réunit au château de Bellevue à Berlin les trois chefs de partis, Angela Merkel pour la CDU, Horst Seehofer pour la CSU et Martin Schulz pour le SPD. Il fit valoir qu'il fallait absolument éviter la constitution d'un gouvernement minoritaire ou la tenue de nouvelles élections anticipées susceptibles de favoriser la poursuite des progrès de l'extrême droite. L'argument était sensible pour les sociaux-démocrates, qui pouvaient craindre dans ce cas d'être dépassés sur le plan national par l'AfD. En fait, au terme de huit heures de débat dans la nuit du 23 au 24 novembre, la direction du SPD avait déjà accepté le principe de discussions exploratoires avec les conservateurs, mais il s'agissait pour elle de faire accepter ce revirement à 180 degrés par la base militante du parti ${ }^{(35)}$. La situation était douloureuse pour les sociaux-démocrates et avivait singulièrement les tensions internes: Stephan Weil, ministre-président du Land de Basse-Saxe qui venait de constituer une grande coalition à la suite des élections régionales, plaidait pour l'acceptation qui se heurtait à l'opposition frontale de l'aile gauche et des jeunes socialistes $(J u s o s)^{(36)}$. Le contexte était d'autant plus délicat qu'à l'extérieur les chrétiensdémocrates ne semblaient guère décidés à transiger avec ce que Horst Seehofer appelait les « revendications démesurées» du SPD. En Bavière, le 4 décembre, ce dernier fut d'ailleurs contraint d'accepter de céder le leadership du parti à son rival Markus Söder dans la perspective des élections régionales de l'automne $2018^{(37)}$.

Réunis en congrès à Berlin le 7 décembre, les sociaux-démocrates acceptèrent finalement la proposition de leur président d'engager des pourparlers avec l'Union, dont l'issue devait rester "ouverte» ${ }^{(38)}$. Retenant la leçon de l'échec des précédentes négociations au sein de la coalition jamaïcaine, les négociations se déroulèrent cette fois-ci dans le huis clos du siège du SPD. En début de matinée, le vendredi 12 janvier 2018, au terme de cinq jours de négociations, les partenaires tombèrent d'accord sur un canevas de contrat de coalition de 28 pages qui reprenait les positions des conservateurs sur la politique migratoire et la fiscalité sur les hauts revenus, avec des contreparties sur les retraites et des avancées en matière d'éducation impliquant une plus grande intervention de l'État fédéral dans le domaine de la compétence exclusive des änder $^{(39)}$. Mais

35 Cécile Boutelet, "Le SPD se résout à une nouvelle grande coalition", Le Monde du 25 novembre 2017; Thomas WIEDER, «Le SPD se veut offensif avant d'aider Merkel à sortir de l'ornière», Le Monde du 28 novembre 2017.

36 Thomas WIEDER, «L'Allemagne avance à reculons vers la grande coalition», Le Monde des 3 et 4 décembre 2017.

37 Thomas WIEDER, «En Bavière, la CSU choisit un nouveau chef très à droite», Le Monde du 6 décembre 2017.

38 Thomas Wieder, «Martin Schulz réélu président d’un SPD divisé », Le Monde du 9 décembre 2017.

39 Thomas WIEDER, «Accord entre CDU et SPD pour diriger l'Allemagne», Le Monde du 13 janvier 2018. 
lors du deuxième congrès extraordinaire du SPD à Bonn le 21 janvier, la poursuite des négociations avec l'Union ne fut adoptée qu'à l'issue de deux tours de scrutin par 362 voix contre 279. Martin Schulz ne fut sauvé que par l'intervention d'Andrea Nahles ${ }^{(40)}$, alors qu'un vote négatif du SPD deux mois après la rupture des négociations au sein de la coalition jamaïcaine aurait fortement mis en péril l'avenir politique d'Angela Merkel. Le mercredi 7 février, les partenaires présentèrent à la presse le contrat de coalition, dont l'adoption définitive restait cependant soumise au vote des 463723 adhérents du SPD. Le SPD avait obtenu trois ministères clés, les Finances, les Affaires étrangères, le Travail et les Affaires sociales ${ }^{(41)}$. Mais Martin Schulz devait être la première victime de ce compromis par défaut: le 7 février, il renonça officiellement à la présidence du parti tout en annonçant son entrée au gouvernement au poste de ministre des Affaires étrangères, entrée à laquelle il dut également renoncer deux jours plus tard le 9 février. Ce double renoncement était lié à l'incompréhension des militants, qui devaient encore se prononcer sur le contrat de coalition, devant le volte-face d'un président qui avait incarné le refus d'une nouvelle grande coalition. Par ailleurs, en revendiquant les Affaires étrangères, Martin Schulz en écartait Sigmar Gabriel qui avait pourtant accepté un an plus tôt de s'effacer à son profit ${ }^{(42)}$. L'échec de Martin Schulz fut total lorsqu'il dut se résigner à ce qu'Andrea Nahles ne lui succède pas comme présidente intérimaire du SPD, au profit d'Olaf Scholz, maire de Hambourg, pressenti par ailleurs comme futur ministre des Finances ${ }^{(43)}$.

Au sein de l'Union l'autorité déclinante d'Angela Merkel était confrontée à l'émergence d'une nouvelle génération de quadras qui ne cachaient plus leur impatience. L'hypothèse d'un départ de la chancelière ne constituait plus un sujet tabou ${ }^{(44)}$, même si ses opposants de premier plan se gardaient bien de contester encore ouvertement sa légitimité sachant les risques encourus. Mais, face à un mouvement d'impatience, la chancelière savait aussi la nécessité qu'il y avait à composer avec ses opposants internes, qui cachaient mal en particulier leur mécontentement devant l'abandon du poste de ministre des Finances au SPD. Le contrat de coalition ne réservait que cinq ministères clés à la CDU et les titulaires des postes de l'Économie et de la Défense restant en place, Angela Merkel ne disposait en fait que d'une marge de négociation réduite, n'ayant que trois ministères à répartir. Le 19 février, Angela Merkel fit désigner Annegret KrampKarrenbauer, "AKK», dans la fonction de secrétaire générale de la CDU. En adoubant la fidèle ministre-présidente de la Sarre comme successeur la plus légitime, la chancelière visait avant tout à sécuriser son propre maintien au pouvoir ${ }^{(45)}$. Le 25 février, elle officialisa sa décision de confier à Jens Spahn, son principal détracteur à l’intérieur de

40 Cécile Boutelet, «En Allemagne, le SPD désuni offre un répit à Merkel», Le Monde du 23 janvier 2018.

41 Thomas Wieder, «Un accord de coalition enfin trouvé en Allemagne», Le Monde du 8 février 2018. Thomas WIEDER, «Une grande coalition dans la douleur en Allemagne», Le Monde du 9 février 2018; Thomas Wieder, «Les jeunes du SPD ne croient pas à la coalition», Le Monde des 11 et 12 février 2018.

43 Thomas Wieder, "Martin Schultz quitte la présidence du SPD dans une ambiance de crise», Le Monde du 13 février 2018.

44 Thomas Wieder, «À la CDU, l'après-Merkel n'est plus tabou», Le Monde du 9 janvier 2018.

45 Thomas Wieder, "Angela Merkel adoube sa dauphine à la tête de la CDU», Le Monde du 21 février 2018. 
la CDU avec le ministre-président du Schleswig-Holstein Daniel Günther, le ministère fédéral de la Santé. Les nominations de Julia Klöckner à l'Agriculture et de Anja Karliczek à l'Éducation participèrent de la même logique générationnelle ${ }^{(46)}$ à la veille du congrès de la CDU à Berlin qui adopta à $97 \%$ des votants le choix d'une nouvelle grande coalition avec le SPD.

Mais l'avenir immédiat d'Angela Merkel restait tributaire du vote a priori incertain des militants sociaux-démocrates partagés entre le désarroi et la colère. Nombreux dans les rangs du parti étaient ceux qui pensaient, au regard de l'érosion continue de son électorat depuis plus d'une décennie, que celui-ci se condamnait au pire en renouvelant son alliance avec les conservateurs. La crise avait fait voler en éclat les sociabilités ouvrières traditionnelles, au fondement des valeurs du parti des "petites gens ». Le SPD se devait d'inventer un nouveau contrat social renouvelant la défense de la valeur d'égalité mais prenant aussi en considération l'exigence de sécurité. La voie d'une nouvelle grande coalition était ouvertement rejetée par les Jusos. Kevin Kühnert, le président des Jusos, incitait les sociaux-démocrates à rompre avec la voie du néolibéralisme, ouverte en 1999 par la fameuse «troisième voie» de Gerhard Schröder ${ }^{(47)}$ et Tony Blair, qui faisait la part belle au libre marché :

«La social-démocratie doit trouver le courage de transformer ses valeurs intemporelles l'égalité, la liberté et la solidarité - et une confiance en soi suffisante pour les faire entrer dans une ère nouvelle. La pusillanimité des années passées a ouvert des failles que les populistes de droite, entre autres, ont utilisées habilement ${ }^{(48)}$.

Kevin Kühnert traduisait bien le sentiment de nombreux militants du parti selon lequel celui-ci continuait de payer chèrement la facture des réformes Hartz de l'ère Schröder. Mais l'appel au sursaut lancé par l'aile gauche et les Jusos était contrebalancé chez les militants par la crainte de voir l'AfD dépasser le SPD en cas de nouvelles élections anticipées. Selon la formule d'un universitaire social-démocrate, le choix se situait «entre la mort lente et la mort rapide» ${ }^{(49)}$. Le dimanche 4 mars 2018 , les résultats du vote des militants sociaux-démocrates furent rendus publics : 66,02\% d'entre eux avaient approuvé le contrat de coalition avec les partis de l'Union. Ainsi prit fin l'interminable avènement de la quatrième grande coalition après six mois de crise.

Le 14 mars 2018, Angela Merkel fut reconduite pour un quatrième mandat par 364 voix, soit 9 de plus que la majorité requise et 35 de moins que sa majorité théorique de 399 élus ${ }^{(50)}$. Second choix pour la chancelière et douloureux mariage de raison pour le SPD, la troisième grande coalition de l'ère Merkel apparaît surtout comme une alliance par défaut. Mais l'institutionnalisation de la grande coalition restera une caractéristique sinon un legs de l'ère Merkel, en contradiction avec le choix effectué en 1949 par Konrad Adenauer. Celui-ci avait alors choisi de promouvoir une démocratie

46 Thomas Wieder, «Merkel rajeunit son équipe pour résister au dégagisme», Le Monde du 27 février 2018.

47 En Allemagne Schröder avait avancé le slogan du « Nouveau Centre».

48 Kevin KüHnert, «Sociaux-démocrates: renoncez au néolibéralisme», Le Monde du 6 avril 2018.

49 Thomas Wieder, «SPD, le grand désarroi», Le Monde du 2 mars 2018.

50 https://www.francetvinfo.fr/monde/europe/allemagne/allemagne-au-pouvoir-depuis-2005-angelamerkel-est-officiellement-reelue-chanceliere-pour-un-4e-mandat_2656060.html 
inspirée du modèle britannique dans laquelle deux grands partis, aux options idéologiques clairement affirmées et tranchées, devaient alterner au pouvoir. Avec Angela Merkel s'est au contraire banalisée, non sans difficultés, une pratique proche du contre-modèle autrichien de la Proporz, celle du partage simultané du pouvoir entre les deux grands partis populaires, qui pose inévitablement la question de la relève des équipes au pouvoir et des modalités de l'alternance. Mais l'affaiblissement des partenaires fait qu'il est difficile aujourd'hui de prévoir si la nouvelle grande coalition arrivera au terme de la législature avec des partis populaires que ne rassemblent plus aujourd'hui qu'à peine un électeur allemand sur deux et auxquels le peuple fait en quelque sorte de plus en plus défaut.

\section{Résumé}

Après une campagne électorale sans éclat dont le résultat ne laissait a priori subsister aucun doute, les élections législatives allemandes du 24 septembre 2017 ont enregistré le très net recul des partis populaires associés dans la grande coalition sortante, plus important encore pour les chrétiens-démocrates que pour les sociaux-démocrates. Dans un paysage politique éclaté, avec la percée de l'extrême droite et le retour des libéraux-démocrates au Bundestag, le visage de la future coalition gouvernementale est apparu d'emblée incertain. Au terme d'une crise sans précédent de six mois, la reconduction d'une nouvelle grande coalition apparaît comme un fragile pis-aller qui n'occulte ni l'affaiblissement de la chancelière, ni le profond désarroi de ses partenaires sociaux-démocrates.

\section{Zusammenfassung}

Nach einer glanzlosen Wahlkampagne, deren Ergebnis a priori nicht den geringsten Zweifel bestehen ließ, haben die deutschen Bundestagswahlen vom 24. September 2017 den sehr deutlichen Rückgang der beiden Volksparteien verzeichnet, die an der ausscheidenden Großen Koalition beteiligt waren. Allerdings wird dabei ein bedeutenderer Rückgang für die Christdemokraten verzeichnet als für die Sozialdemokraten. In einer zersplitterten politischen Landschaft, in der sowohl der Durchbruch der rechtsradikalen Parteien als auch die Rückkehr der Freidemokraten in den Bundestag hervortreten, bekam das Gesicht der zukünftigen Regierungskoalition auf Anhieb immerhin unfeste Umrisse. Nach Ablauf einer beispiellosen sechsmonatigen Krise kommt die Fortsetzung einer neuen Großen Koalition wie eine notdürftige Lösung vor, die weder die Schwächung der Kanzlerin noch die tiefe Verwirrung ihrer sozialdemokratischen Koalitionspartner zu vertuschen vermag. 